\title{
EL OURIL COMO EJEMPLO DEL USO DE LOS JUEGOS CULTURALES PARA LA ENSEÑANZA GLOBALIZADORA DE LAS MATEMÁTICAS
}

Espinar Mesa, Georgina; Fernández-Oliveras, Alicia; Oliveras Contreras, María Luisa. Universidad de Granada.

Fecha de recepción: 30 de junio de 2014.

Fecha de revisión: 5 de julio de 2014.

Fecha de aceptación: 15 de julio de 2014.

\section{Resumen}

En este artículo se describe el proceso de elaboración de una propuesta didáctica para educación infantil, enmarcada en el ámbito del aprendizaje basado en proyectos. El punto de partida fue la realización de una investigación teórica educativa que indagaba en la integración de las Etnomatemáticas en las aulas y en uso del juego como recurso educativo. La investigación realizada condujo a la elaboración de la propuesta didáctica siguiendo un modelo de proyecto educativo denominado "microproyecto". El microproyecto se diseñó a partir de un signo cultural típico de Cabo Verde: el juego del Ouril. Mediante este microproyecto se pretende trabajar aspectos de las matemáticas desde un enfoque globalizado, incorporando signos característicos de otras culturas y con el juego como eje central.

Palabras clave: Constructivismo social, matemáticas, educación intercultural, aprendizaje basado en proyectos, estrategia globalizadora, formación de profesores.

Nos encontramos en una época culturalmente muy diversa. Este marco sociocultural en el que nos encontramos, hace que, como futuros educadores y educadoras, nos planteemos nuevos retos de futuro; entre los cuales estará el buscar modelos educativos y de convivencia interculturales, que hagan posible una cohesión social. Si queremos aplicar un modelo intercultural, la mejor manera será plantearnos objetivos en nuestros retos como los de compartir con reciprocidad elementos lingüísticos, culturales y simbólicos. Todos ellos los podemos llevar a cabo mediante la educación de una forma globalizada, es decir, incluyendo todas las áreas y tomando la lógicamatemática como eje central, ya que así se trabajan las estructuras de pensamiento mediante las cuales se integrarán todos los conocimientos de una manera más sólida.

Hay que interpretar las matemáticas como un recurso que nos sirve de fuente de conocimiento y para comprender el mundo y la sociedad en la que vivimos. En nuestra vida diaria siempre pensamos matemáticamente, para el desempeño de funciones diarias, jugar, etc. Es por ello que el conocimiento matemático es tan importante y necesario. Dado que es muy importante la relación del pensamiento informal y el pensamiento formal, se deben crear puentes para relacionar las ideas con las actividades, partiendo de lo concreto, de situaciones cercanas a los niños y niñas, para comenzar a construir conceptos y relaciones, y pasar al pensamiento abstracto. Hay situaciones, materiales, (monedas, juego, etc.) que forman parte de la vida diaria del niño/a que pueden servir para fomentar el pensamiento abstracto. Por ello desarrollaremos una propuesta didáctica, con un enfoque basado en el método de 
"Proyectos", y su adaptación en forma de "Microproyectos" realizada por Oliveras (1996).

Con este microproyecto pretendemos trabajar las matemáticas a partir del juego denominado "Ouril" y desde una metodología donde prime la manipulación y la experimentación favoreciendo así las capacidades básicas de niños y niñas de edades comprendidas entre 5 y 6 años. Las actividades relacionadas con el juego Ouril están pensadas para dar a los niños y niñas diferentes estrategias que puedan aplicar en diferentes situaciones, reales y prácticas. Con las actividades de este microproyecto intentamos que los alumnos/as pasen del pensamiento concreto al abstracto mediante el juego, ya que este es un material cercano a ellos/as y que permite el desarrollo de todas las áreas de conocimiento, favoreciendo el logro de los objetivos propios del segundo ciclo de la Educación Infantil.

\section{El juego Ouril y su contexto}

Nos hemos centrado en un objeto de estudio relevante para la cultura de Cabo Verde: el Ouril, un juego tradicional en esa zona. La primera isla de Cabo Verde donde apareció este juego fue Santiago y de allí se extendió a las demás islas.

El archipiélago del Océano Atlántico que compone Cabo Verde está ubicado al oeste del continente africano, cerca de Senegal. Republica de Cabo Verde es el nombre oficial de esta antigua colonia portuguesa, formada por diez islas principales y cinco menores, que juntas forman una $\mathrm{C}$, y cuya capital es Praia. Las islas son de origen volcánico y fueron descubiertas en el siglo XV por los portugueses, quienes las habitaron y convirtieron tristemente en un centro de trata de esclavos. A consecuencia de esto, la mayor parte de los habitantes son mezcla de esclavos africanos y colonizadores europeos. Su lengua oficial es el portugués aunque la población mayoritariamente habla una lengua propia de tradición oral, denominada criollo caboverdiano (Crioulo).

La bandera oficial de Cabo Verde se compone de cinco rectángulos longitudinales. Los rectángulos superior e inferior son de mayor tamaño y de color azul, simbolizando el océano y el cielo. Esos dos rectángulos se separan con los otros tres, de igual tamaño y de color blanco rojo y blanco respectivamente, encarnado la paz y el esfuerzo. En el lateral derecho de la bandera hay un circulo con diez estrellas de cinco puntas amarillas, que representan las diez islas mayores.

El Ouril (también conocido como uril, oril, ouri, ori, etc) pertenece a la familia de los juegos "Mancala" y es uno de los juegos de tablero con más antigüedad. Requiere un tablero, llamado oware, y unas fichas que son semillas llamadas ouris, procedentes de los arbustos conocidos como ourinzeira. El tablero es una tabla rectangular que posee dos hileras con seis agujeros circulares. Las piezas son, en total, 48 semillas (24 por jugador) y se disponen inicialmente de forma que haya 4 semillas en cada agujero del tablero. La dirección siembra es anti-horaria. El objetivo del juego es sembrar y cosechar, capturando más semillas que el adversario.

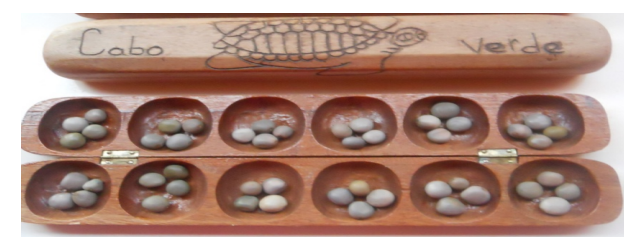

Figura 1. Imagen de un Ouril caboverdiano 
Este juego fue llevado por los esclavos durante el colonialismo portugués, es por ello que es más habitual ver jugar a hombres que a mujeres. El Ouril también se juega en Portugal.

Hemos elegido este objeto de estudio porque es una forma de acercar las diferentes culturas y fomentar el desarrollo global en la edad infantil desde una forma cercana a ellos, a su mundo, como es la parte lúdica.

\section{Microproyectos}

Microproyecto es un plan de trabajo o proyecto educativo, interdisciplinar en cuanto a los contenidos y abierto en los objetivos. Desarrolla el autoaprendizaje y trabajo en pequeños grupos, en el cual el rol del profesor es de dinamizador y partícipe de los descubrimientos y de coordinador de las interacciones. Incluirá un objeto de estudio, procurando que sea un objeto/artefacto cultural, que permita ser analizado desde los diversos puntos de vista de las distintas ciencias, y a la vez, el conocimiento obtenido será vehiculado mediante los distintos lenguajes y expresiones artísticas. En suma se trata de un proyecto, en el plano educativo, que permite acceder a una pequeña parte de la cultura con sentido en sí misma, y que al ser estudiado desde distintos puntos de vista científicos provoque aprendizajes contextualizados con significado dentro del mundo infantil. Se trata de una metodología activa y de unos recursos globalizados que, elaborados desde una perspectiva didáctica socio-constructivista, hacen que en su puesta en práctica se generen significados matemáticos contextualizados. El microproyecto también recogerá objetivos de otras áreas, integrando el aprendizaje global del niño. Para desarrollar esta propuesta de microproyecto partimos de unos fundamentos epistemológicos y didácticos, que indican el punto de vista sobre la educación, el juego y las matemáticas desde el cual hacemos tal propuesta (Oliveras 2005).

\section{Etnomatemáticas}

Llamaremos Etnomatemáticas a las matemáticas que son practicadas por grupos culturalmente identificables, como son sociedades nacionales o tribales, grupos y oficios, niños de un cierto intervalo de edad, clases profesionales, incluyendo sus jergas y modos de expresión. Este concepto de "Etno" incluye a todos los grupos identificables culturalmente con sus jergas, códigos, símbolos, mitos, y sus procesos específicos de razonamiento e inferencia (D'Ambrosio, 1985).

Bishop (1988, p.182) mencionó la relación de las matemáticas con las prácticas sociales: "existen seis actividades humanas universales practicadas de modos diferentes en cada cultura: contar, medir, localizar, dibujar, explicar y jugar".

Según Mtetwa (1995), algunas personas han interpretado mal este término, usándolo exclusivamente para designar, a las formas matemáticas creadas y practicadas por y para un grupo "étnico" específico. Este concepto de etno corresponde al concepto de etnociencia establecido por antropólogos y etnógrafos del siglo XIX con mentalidad colonialista que constituye un reduccionismo de tipo positivista, en el cual existe, por una parte, la ciencia establecida en el ámbito de las potencias político-geográfico dominante, conocimientos infra calificados como "etno" (Mtetwa, 1995).

No compartimos esta visión de los fenómenos culturales, sino otra, con otro sentido o enfoque, en el que la interpretación de la vida científica y social de cualquier cultura se ejecuta mediante sus propios elementos culturales. Con criterios ecológicos o contextualizados que conllevan una valoración de equidad ontológica, independiente 
de superioridades racionalistas, mapas o dominaciones.

Las Etnomatematicas representan al conjunto de todas las matemáticas existentes, en tanto que consideramos a esta ciencia como un conjunto de prácticas específicas, realizadas por diversos tipos de personas, comunidades, para el desempeño de cometidos diferentes que satisfacen sus intereses producidos como grupo. Es decir prácticas prototípicas que constituyen parte importante y representativa de culturas y microculturas diferentes (Oliveras, 1996).

\section{El juego}

El juego ha estado presente a lo largo de toda la historia y en todas las culturas, pero presenta diferentes características en función de su contexto cultural. El juego es una de las actividades básicas de la infancia, indispensable para el desarrollo psicomotor, cognitivo, afectivo y social de los niños y niñas.

El juego es un recurso didáctico de un gran valor educativo, además está reconocido como derecho fundamental de la infancia.

El juego puede definirse como una actividad necesaria para los seres humanos y de gran valor en la esfera social, ya que permite ensayar ciertas conductas sociales, a su vez es un recurso útil para adquirir y desarrollar capacidades cognitivas, motrices o afectivas. Esta actividad se debe practicar sin ningún tipo de obligación, con el tiempo y espacio necesario.

Linaza (1991) establece, en los juegos y actividades lúdicas, una serie de criterios, para poder identificarlos y caracterizarlos:

- El juego es libre, si es una imposición externa no es un juego, esta condición ha de ser libre. Entendiendo entonces que el juego ha de ser voluntario y libre, ya que como comentaba Boule, una persona jugará más si esta quiere, y durante el tiempo que esta quiera.

- No estará condicionado por refuerzos o acontecimientos externos. No se juega para tener una consecuencia posterior (reconocimiento social, juicio externo,...), si la hay no se trata de actividad lúdica.

- El juego produce placer, tiene un papel muy importante ya que hace que el niño exprese libremente sus impulsos y de una manera aceptada, esto hace que se reduzcan sus miedos y ansiedades.

- Predominan los medios sobre los fines, es el rasgo más característico del juego, ya que lo que produce la propia acción de jugar, es la satisfacción de realizarlo. El objetivo principal del juego son las propias acciones.

- Las conductas lúdicas presentan ciertas especificidades, siempre se pone de manifiesto el carácter de ficción.

Teniendo en cuenta que el juego tiene que ser libre y espontáneo, debería ser el niño quien decida cuando jugar, esto no quiere decir que el juego en el aula no se pueda practicar, o sea escaso; pero lo que el maestro debe hacer es presentar actividades con carácter lúdico y con la práctica cotidiana el niño se percatará de la intencionalidad de estas.

Según la clasificación de juegos que hacia Piaget (1961), el Ouril estaría dentro de los juegos de reglas; lo que se pretende con este tipo de juego, es dominar del egocentrismo mediante la socialización y la competición. El alumno tiene que aceptar distintas normas, incluso llegar a consensuarlas con sus compañeros, o de ponerse en 
el lugar de su rival para prever su victoria, es las razones de que este tipo de juego tenga un gran valor didáctico.

Para que un juego utilizado en el aula sea considerado de estrategia más que de azar, este deberá tener una serie de requisitos:

- Tiene una serie de reglas fijas, definidas al principio de la partida.

- Las reglas dejan claro los objetivos de todos los jugadores/as.

- Los jugadores/as eligen las acciones para alcanzar su objetivo. Si no es así es un juego de azar.

- Las reglas fijan cuando termina el juego, expresando un vencedor/a y un perdedor/a.

Algunos maestros/as no creen que el uso de este tipo de juegos en el aula pueda ser positivo ya que este tipo de juegos presentan un vencedor/a, un perdedor/a o empate. Pero se ha de tener en cuenta que la competición puede presentar utilidades didácticas si se gestiona adecuadamente. Según Kamii y Devries (1988) los niños tienen tal egocentrismo que les impide centrarse en lo que hacen los demás. Además la competición en el juego no es para obtener un refuerzo externo, hay que centrarlo en la acción; ya que sino muchos alumnos perseguirían la recompensa y no se realizaría el aprendizaje.

Se debe dejar claro que el vencedor/a solo ha ganado un juego, quitándole importancia al hecho de ganar; el hecho de perder también forma parte del juego y no por ello se es inferior, ni trae consigo algún tipo de consecuencia; hay que permitir a los alumnos/as que eviten la competición si no quieren; la posibilidad de ganar en los juegos de estrategia son puramente de habilidad y esto puede hacer que ciertos jugadores dejen de jugar si siempre pierden, si vemos que esto ocurre, lo que debemos hacer es ir incorporando el azar en el juego.

\section{MARCO LEGISLATIVO}

Mostraremos un breve recorrido histórico sobre el marco legislativo educativo y se verá la concepción de juego en las diferentes leyes.

- L.O.G.S.E. (Ley Orgánica 1/1990, de 3 de octubre de 1990, de Ordenación General del Sistema Educativo). Da definitivamente un carácter educativo a la educación infantil, establece los aspectos básicos del currículo y denomina explícitamente la necesidad de una metodología basada en el juego para el desarrollo de las capacidades básicas del niño y niña." la metodología educativa se basará en experiencias, actividades y juego, en un ambiente de afecto y confianza". (Art. 9.5.).

- L.O.C.E. (Ley Orgánica 10/2002, de 23 de diciembre, de Calidad de la Educación). Esta ley mantiene la metodología basada en el juego durante la etapa infantil, aunque implica grandes cambios. Supone educativa la etapa 3-6 y deja fuera del sistema educativo la franja de 0-3 considerándola asistencial. Por cambios políticos esta ley no ha llegado a llevarse a cabo.

- L.O.E. (Ley Orgánica 2/2006, de 3 de mayo, de Educación). Vuelve a los planteamientos de la LOGSE recuperando así el carácter educativo. Considera el juego como recurso metodológico para la institución educativa durante la etapa de infantil y la actividad lúdica como una necesidad básica de la infancia. En cuanto a la metodología de intervención, menciona: "Los métodos de trabajo en ambos ciclos se basarán en las experiencias, las actividades y el juego y se aplicaran en un ambiente de afecto y confianza, para poder potenciar la autoestima y la 
integración social" (Art. 14.6). Esta concepción educativa del juego queda reflejada a lo largo del despliegue legislativo de los últimos años.

- ORDEN de 5 de agosto de 2008 por la que se desarrolla el Currículo correspondiente a la Educación Infantil en Andalucía. Menciona: "El juego, instrumento privilegiado de intervención educativa (...) suele suponer para el niño situaciones placenteras (...) Estas características hacen que el juego afecte al desarrollo afectivo, psicomotor, social, cognitivo y lingüístico, de ahí su importancia para un crecimiento global y armónico. A través de los juegos, niñas y niños se aproximan al conocimiento del medio que les rodea, al pensamiento y a las emociones propias y de los demás." (Apartado C. 3).

\section{FUNDAMENTOS DIDÁCTICOS}

Para llevar a cabo este microproyecto nos basamos en las teorías pedagógicas de Piaget y Vygotski, es decir en una metodología constructivista, ya que consideramos que es un enfoque en el que la experimentación y la interacción con el medio y social, es un factor primordial para la construcción y el desarrollo de la propia inteligencia.

Piaget y Vygotski se apoyan en un enfoque constructivista social para el aprendizaje. Defienden la idea que el individuo construye su propio comportamiento, tanto en aspectos cognitivos como sociales y afectivos, como resultado de la interacción entre la experiencia y la toma de decisiones.

Según Piaget (citado por Ruiz, 2005): “El aprendizaje se apoya en la acción”. Mediante las experiencias el individuo va organizando, estructurando y reestructurando los esquemas previos del pensamiento, modificándolos y enriqueciéndolos. Todo esto se da gracias a estas experiencias; pero debemos tener en cuenta que estas experiencias se llevan a cabo mediante la interacción con el mundo físico y social; como afirmaba Vygotski (1978), en la idea básica de la psicología social, los conflictos cognitivos entre miembros de un mismo grupo social pueden facilitar la adquisición de conocimientos. De esta forma iremos construyendo nuestra propia inteligencia. Realizaremos esta construcción, gracias a los esquemas que los individuos ya poseen y poniéndolos en relación con el medio que lo envuelve y actividades constructivistas.

\section{PROGRAMACIÓN DEL MICROPROYECTO}

\section{Objetivos generales}

Tomamos los objetivos del BOJA que guardan relación con el objeto de estudio, como:

- Construir su propia identidad e ir formándose una imagen positiva y ajustada de sí mismo, tomando gradualmente conciencia de sus emociones y sentimientos a través del conocimiento y valoración de las características propias, sus posibilidades y límites.

- Establecer relaciones sociales satisfactorias en ámbitos cada vez más amplios, teniendo en cuenta las emociones, sentimientos y puntos de vista de los demás, así como adquirir gradualmente pautas de convivencia y estrategias en la resolución pacífica de conflictos.

- Observar y explorar su entorno físico, natural, social y cultural, generando interpretaciones de algunos fenómenos y hechos significativos para conocer y comprender la realidad y participar en ella de forma crítica.

- Comprender y representar algunas nociones y relaciones lógicas y matemáticas referidas a situaciones de la vida cotidiana, acercándose a estrategias de resolución de problemas. Debe contribuirse, igualmente, a que los niños y las 
niñas constaten la existencia en nuestras vidas de situaciones con interrogantes o incógnitas cuya resolución exige la reflexión sobre ellas y la aplicación de esquemas de pensamiento. El acercamiento a la resolución de problemas propios del contexto en el que se vive, descubriendo y utilizando algunas de las estrategias que para ello podemos emplear, es otra de las intencionalidades que se pretenden.

- Utilizar el lenguaje oral de forma cada vez más adecuada a las diferentes situaciones de comunicación para comprender y ser comprendido por los otros.

- Conocer y participar en algunas manifestaciones culturales y artísticas de su entorno, teniendo en cuenta su diversidad y desarrollando actitudes de interés, aprecio y respeto hacia la cultura andaluza y la pluralidad cultural.

\section{Áreas de Educación Infantil}

- Ser progresivamente autónomo/a en la realización de actividades.

- Realizar movimientos globales y segmentarios y adoptar diferentes posturas, y se sitúa en el espacio atendiendo a las nociones delante-detrás, un lado-otro lado e izquierda.

- Progresar en la coordinación dinámica general y óculo-manual.

- Desarrollar hábitos de respeto, ayuda y colaboración.

- Participar en el juego.

- Establecer relaciones respetuosas, afectivas y recíprocas con niños y niñas, aceptando diferencias.

- Adquirir algunas nociones de Geografía y de Historia a través de los relatos contados por el docente sobre otros lugares y otras culturas.

- Conocer los roles y responsabilidades de las personas de otros tiempos.

- Descubrir las costumbres y la forma de vida de personas de otros pueblos y culturas.

- Conocer y aceptar las normas que hacen posible la vida en grupo y el trabajo en equipo.

- Discriminar e identificar comportamientos adecuados e inadecuados para la convivencia.

- Reforzar contenidos matemáticos aprendidos.

- Compone y descompone números hasta el 9.

- Resuelve operaciones matemáticas sencillas.

- Agrupa y clasifica objetos atendiendo al color, a la forma y al tamaño, diferencia la cualidad de los objetos.

- Nombra medios de transporte y utiliza el vocabulario nuevo aprendido.

- Escuchar atentamente las historias contadas por el docente sobre otros pueblos del mundo para comprender la información y ampliar el vocabulario.

- Escuchar, preguntar, pedir explicaciones y aclaraciones, y aceptar las orientaciones dadas por el docente.

\section{Contenidos}

- Aceptación de la identidad y características de los demás.

- Sentimientos y emociones.

- Ayuda y colaboración con sus compañeros y compañeras.

- Habilidades para la interacción y la colaboración: trabajo en equipo.

- Coordinación dinámica general y coordinación óculo-manual.

- Control del movimiento en los desplazamientos.

- Aceptación de las normas que rigen el juego.

- Desarrollo de la observación y de la atención. 
- Percepción de semejanzas y diferencias.

- Iniciación a la Geografía y a la Historia.

- Percepción de diferentes tamaños: el más largo, cortos, etc

- Nociones espaciales: izquierda-derecha.

- Conteo.

- Series.

- Lectura y asociación de imágenes.

- Lectura e interpretación de etiquetas.

- Vocabulario básico: Ouril, semillas, cosecha, etc

- Técnicas plásticas: colorear, pegar...

- Escucha atenta a las historias contadas por el docente.

- Visualización de imágenes y fotografías sobre el juego del Ouril y Cabo Verde

\section{Actividades}

Se proponen actividades en torno al juego del Ouril para realizar en el aula y alcanzar los objetivos propuestos. Estas actividades son:

\section{Contamos un cuento}

El/la docente presentará en la primera actividad un cuento en el que aparezca el juego del Ouril. Después de escucharlo y comentarlo, mediante la asamblea y el debate hará una serie de preguntas para que el alumnado se motive hacia el tema en cuestión.

$\left.1^{\circ}\right)$ Estas preguntas serán: ¿Sabéis qué es el juego del Ouril? ¿Quién juega al juego del Ouril?¿Cómo creéis que es un Ouril?¿Cuántas personas pueden jugar a este juego?¿De dónde proviene este juego?

$2^{\circ}$ ) Seguidamente buscaremos en Internet el juego del Ouril y contrastaremos nuestras respuestas.

$\left.3^{\circ}\right)$ Escribiremos en la pizarra: "Ouril", para que el alumnado se familiarice con el nombre.

$4^{\circ}$ ) Los alumnos y alumnas dibujarán un Ouril, escribirán su nombre y al lado un harán lo miso con un juego típico de su propia cultura.

Metodología: En esta actividad el profesor/a hará de mediador de las conversaciones que surjan en la asamblea, realizando preguntas e interviniendo cuando sea necesario.

Recursos: Cuento, la asamblea, fotografía del juego Ouril, folios y lápices de colores

Criterios y elementos a observar para la evaluación: Los conocimientos previos, el vocabulario, la expresión oral y escrita.

\section{Localizamos en el mapa Europa y Cabo Verde}

Al final de la primera actividad pasaremos hacer una tarea de medida con distintos materiales.

$1^{\circ}$ ) Presentaremos el mapamundi y, partiendo de la base de que estámos trabajando diferentes continentes y culturas, nos ubicamos en Europa inicialmente. 
$2^{\circ}$ ) Después enseñaremos donde está Cabo Verde, y haremos un debate sobre la distancia, los medios de transporte con lo que se pueden acceder, etc.

$3^{\circ}$ ) Mediremos las distancias. Cada grupo tendrá un "medio de medición" diferente:

- Grupo rojo: rotuladores

- Grupo verde: regletas cousiners ( una de cada)

- Grupo amarillo: con las manos

- Grupo azul: con los pies

$\left.4^{\circ}\right)$ Representaremos las distancias.

En un mural dibujaremos una tabla con las diferentes medidas.

Metodología: El profesor/a tendrá un papel de guía y de mediador.

Recursos: Mapamundi, regletas cuisinier, rotuladores, cartulina.

Criterios y elementos a observar para la evaluación: El profesor observará las reacciones de los niños ante las diferentes situaciones.

En estas dos actividades se trabajan los conocimientos sociales y lógico-matemáticos ya que aparecen normas, convenios, signo, representaciones y lenguajes; además establecen relaciones entre los objetos, sujetos u otras relaciones previas.

\section{Qué es un Ouril}

Llevaremos un Ouril a clase, recordaremos el juego del Ouril que salía en el cuento, comentaremos la historia de este juego, que se basa en la siembra y la cosecha y explicaremos las reglas.

Será una actividad introductoria para acercar los materiales del juego del Ouril. Los niños/as investigaran libremente los distintos materiales y descubrirán nuevas texturas y nuevas formas.

Metodología: Será una actividad que tiene una parte introductoria donde se presenta el material y el profesor/a hace de guía y otra parte libre, donde los niños y niñas investigarán libremente y el/la docente solo intervendrá para resolver dudas.

Recursos: Tablero de Ouril con todos sus materiales.

Criterios y elementos a observar para la evaluación: Cómo interactúan los niños con los diferentes objetos.

En esta actividad se trabajan los conocimientos físicos, sociales y lógicomatemáticos ya que aparecen normas, convenios, signo, representaciones y lenguajes; se captan propiedades de los objetos mediante los sentidos; además establecen relaciones entre los objetos, sujetos $u$ otras relaciones previas.

\section{Construcción de un tablero de Ouril}

Una vez introducidos los conceptos tratados en las actividades anteriores, fabricaremos nuestro Ouril a partir de material reciclado. Para obtenerlo contaremos 
con la ayuda de las familias. Concretamente, para confeccionar los tableros pediremos que lleven al aula hueveras de las que se emplean para dispensar los huevos en los comercios. La actividad se puede realizar individualmente o en pequeño grupo. Repartiremos el material necesario y el alumnado deberá situarlo en el lugar correspondiente, siguiendo los pasos de la elaboración:

1. pintamos la huevera a nuestro gusto con colores de tempera

2. una vez seca, rodeamos los bordes de los agujeros con plastilina

3. repartimos las semillas por equipo.

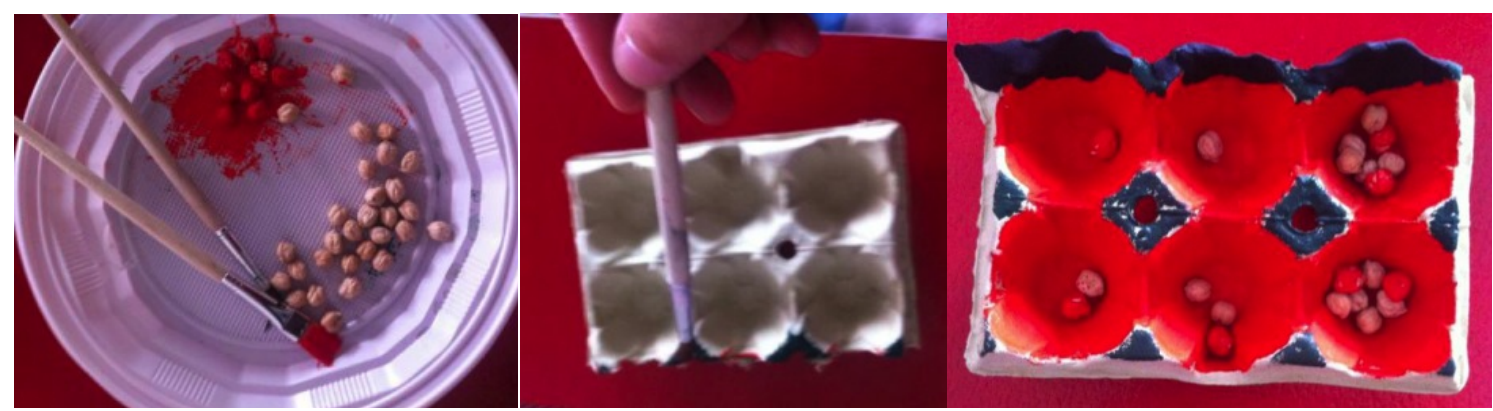

Figura 2. Imágenes relacionadas con la actividad Construcción de un tablero de Ouril.

Metodología: El maestro/a explicará la actividad actuando como guía y cada alumno/a o grupo creará su propio Ouril, organizando los diferentes elementos.

Recursos: Una huevera de media docena, colores de tempera, plastilina, pinceles, 24 semillas (12 de una forma y las 12 de otra distinta).

Criterios y elementos a observar para la evaluación: Se evaluará la creatividad de cada alumno/a, el reconocimiento de nuevas formas geométricas y su orientación espacial.

\section{Jugamos con nuestro Ouril}

Para finalizar, jugaremos con nuestro Ouril. Así podremos tener una vivencia de todas las cosas que hemos trabajado. En primer lugar, nos sentaremos todos juntos y haremos un pequeño recordatorio sobre todo lo visto en estos días y el/la docente aprovechará para contar las reglas del juego. Al finalizar dejaremos que el alumnado juegue de forma libre primero, y después, siguiendo las reglas del juego.

Teniendo en cuenta la dificultad del juego original, hemos hecho una adaptación para los niños/as de esta corta edad.

Objetivo general del juego: Cosechar el mayor número de semillas.

Reglas del juego:

1. Cada jugador/a coloca 4 semillas en cada hoyo, es decir, reparte 12 semillas entre los tres agujeros de su fila. (En total habrá 24 semillas, distribuidas de 4 en 4 en los seis agujeros).

2. En tiradas alternadas, cada jugador/a cogerá todas las semillas de uno de los agujeros de su fila y las repartirá, de una en una, por cada agujero que tiene a la derecha. 
3. Si la última semilla cae en un agujero de su fila, el jugador/a recogerá la cosecha, es decir, se llevará todas las semillas del agujero.

4. Si la última semilla cae en un agujero de la fila contraria, no se llevará ninguna cosecha y le dará paso al jugador/a contraria.

5. Gana el jugador/a que consigue cosechar más semillas.

Metodología: En esta actividad los niños/as tendrán que realizar el juego del Ouril. El maestro/a será quien marcará las normas y resolverá posibles dudas.

Recursos: Nuestro Ouril.

Criterios y elementos a observar para la evaluación: Se evaluara la buena adquisición de las normas y el trabajo cooperativo de los niños y niñas.

En estas dos últimas actividades, al igual que en las dos primeras, se trabajan los conocimientos sociales y lógico-matemáticos.

Con este juego no solo deben buscar la mejor estrategia para vencer, conviene que sepan cumplir las reglas como ser capaz de diferenciar ciertos atributos, aprender a oponerse al adversario, utilice el número para comparar, realice descomposiciones, haga seriaciones, etc. El aprendizaje se da desde el primer momento ya que el alumno/a aprende a colocar las fichas/semillas en sus respectivas posiciones; seguidamente tiene que desarrollar estrategias para avanzar en el juego, si no lo hará mediante la observación de sus iguales, observando los movimientos de sus compañeros y así obtiene más estrategias. Con esta clase de juego también frenamos el egocentrismo muy presente en estas edades.

\section{CONCLUSIONES}

En esta investigación y propuesta didáctica, podemos ver que hay diferentes formas de tratar la diversidad cultural en las aulas de Educación Infantil.

Como ya sabemos, cada vez hay más diversidad cultural, pero con esta propuesta no pretendemos enseñar a tratar a las diferentes culturas, si no a aprender sus métodos para resolver diferentes situaciones, para posteriormente saber aplicarlos a nuestra cultura y así enriquecernos de los diferentes conocimientos culturales.

Con este trabajo se puede observar que, mediante una metodología globalizada y tomando las etnomatemáticas matemática como eje central de la técnica denominada, acercamos las matemáticas de otras culturas a nuestras aulas de Educación Infantil y conseguimos que nuestro alumnado las identifiquen, las conozcan, las vivan y las sepan utilizar en diferentes contextos.

Hemos utilizado un recurso muy familiar a la vez que motivador para los niños y las niñas, como es el juego. Creemos que es un medio que facilita la adquisición de nuevos conceptos y comportamientos, ya que plantea problemas o retos que deben resolverse con unas normas o pautas a seguir. Esto, además de facilitar aprendizajes significativos, hace que se fomente el cooperativismo entre iguales.

\section{BIBLIOGRAFÍA}

Bishop, A. J. (1988). Mathematics education in its cultural context. En A. J. Bishop, (Ed.), Mathematics Education and Culture (pp. 179-192). Dordrecht: Kluwer Academic Publishers. 
D'Ambrosio, U. (1985). Ethnomathematics and its place in the history and pedagogy of mathematics. For the Learning of Mathematics, 5(1), 44-48.

Kamii, C y Devries, R. (1988) Juegos colectivos en la primera enseñanza: implicaciones de la teoría de Piaget. Madrid: Plaza.

Ley Orgánica 1/1990, de 3 de octubre de 1990, de Ordenación General del Sistema Educativo. BOE, 238, 28927-28942.

Ley Orgánica 10/2002, de 23 de diciembre, de Calidad de la Educación. BOE, 307, 45188-45220.

Ley Orgánica 2/2006, de 3 de mayo, de Educación. BOE, 106, 17158-17207.

Linaza, J. L. (1991). Jugar y aprender. Madrid: Alhambra Long-man.

Mtetwa, D. (1995). Matemática y etnomatemática: Punto de vista de los estudiantes de Zimbabwe. Boletín ISGEM, Vol. 7, N 1. En Scott, P. (Ed.), Un compendio de los Boletines del Grupo Internacional de Estudios de Etnomatemáticas. Agosto 1985 a diciembre 1994.

Oliveras, M. L. (1996). Etnomatemáticas. Formación de profesores e innovación curricular. Granada: Comares.

Oliveras, M. L. (2005). Microproyectos para la Educación Intercultural en Europa. Revista UNO, 38, 70-81.

Orden de 5 de agosto de 2008 por la que se desarrolla el Currículo correspondiente a la Educación Infantil en Andalucía. BOJA, 169,17-53.

Piaget, J. (1961). La formación del símbolo en el niño. México: Fondo de cultura económica.

Ruiz, L. (2005). Aprendizaje y matemáticas. La construcción del conocimiento matemático en la Escuela Infantil. En M. D. Chamorro, J. M. Belmonte, M. L. Ruiz y F. Vecino (Ed.), Didáctica de las matemáticas para Educación Infantil (pp. 1-38). Madrid: Pearson.

Vygotski, L. (1978). Mind in Society. Cambridge: Harvard University Press.

\section{WEBGRAFÍA}

História de Cabo Verde extraída de:

http://www.islascaboverde.com/

http://es.wikipedia.org/wiki/Cabo_Verde

Información sobre el juego del Ouril extraída de:

http://www.awale.info/africa-occidental/ouril/?lang=es

http://blocs.mesvilaweb.cat/node/view/id/239297

http://webfacil.tinet.org/jtc/185140 